Pacific Journal of Mathematics

SPECTRAL DECOMPOSITION OF A CLASS OF OPERATORS 


\title{
SPECTRAL DECOMPOSITION OF \\ A CLASS OF OPERATORS
}

\author{
R. C. Sine
}

1. Introduction. Recently there has been a great interest in the study of various classes of operators on a complex Banach space with the purpose of developing a spectral theory generalizing that of selfadjoint operators on Hilbert space. Most well-known is the work of N. Dunford [2,3] on spectral operators. Many operators of interest are ruled out of this class by the requirement that there exist a countably additive resolution of the identity; for example, U. Fixman [5] has given examples of invertible isometries of $L_{p}(p \neq 2)$ and $C(X)$ which fail to be spectral. This present work is directed toward the development of a spectral theory where the resolution of the identity need only be finitely additive.

The general method is to use the classical operational calculus,

$$
\varphi: f \rightarrow f(T)=\frac{1}{2 \pi i} \int_{\gamma} f(z) R(z ; T) d z,
$$

to induce a ring homomorphism of $\mathfrak{F}(T)$, the ring of all functions analytic on neighborhoods of $\sigma(T)$. This calculus may be used to induce a norm on $\mathfrak{F}(T)$ by defining $|f|$ to be $|f(T)|$. We may complete $\mathfrak{F}(T)$ in this norm to obtain a Banach algebra $A$ which includes $\mathfrak{F}(T)$ as a dense subspace and on which there is a unique continuous extension of the classical homomorphism. Alternatively, under certain hypotheses, we may equip $\mathfrak{F}(T)$ with a norm so that the completion in this norm yields a regular function algebra.

What we will need, and therefore hypothesize, is a norm that accomplishes both conditions, namely, the operational calculus, $\varphi$, is to be bounded on $\mathfrak{F}(T)$ and the completion of $\mathfrak{F}(T)$ is to be a regular function algebra. We will need to impose further conditions at the "wrong end" of the theory; it will not be obvious from knowledge of an operator whether these conditions are satisfied. It will be clear, however, that the operationally defined class of operators contains some more easily indentified classes. We also impose a "front end" restriction on the spectrum of the operator, the $R$-set property.

In $\S 2$, we obtain the consequences of the existence of an opera-

Received February 25, 1963. This paper is part of a doctoral dissertation submitted to the University of Illinois written under Professor R. G. Bartle. The author expresses his warmest thanks to Professor Bartle who has given much of his time discussing and criticizing this work. This work was supported in part by a National Science Foundation Fellowship and Grant 19869. 
tional calculus satisfying a certain continuity condition. The theory developed there will encompass: (A) self-adjoint and unitary operators on a Hilbert space; (B) operators of polynomial growth (a generalization of the "weakly almost periodic" operators of E. Lorch [9]); (C) operators of D. Smart [14]; and (D) spectral operators of scalar type which have spectra of a certain class. The main result, Theorem 2.3 , relates the support of a function and the spectrum of a vector.

In $\S 3$, we introduce the $N^{*}$-condition and thereby obtain a spectral decomposition of a class of operators with special attention given to operators of polynomial growth. With the assumption that the spectrum is purely continuous and is contained in the unit interval, a family of closed commuting projections is obtained under the $N^{*}$-condition.

Section 4 is concerned with the problem of the synthesis of an operator from its parts in the form of an integral representation. A decomposition analogous to that of spectral operators is given.

The notation terminology follow that of [4] and [8].

We conclude this present section with a few preliminary definitions and results. These definitions and Theorem 1.1 have been given by Dunford $[2,3]$ for spectral operators.

For each $x$ in $\mathfrak{X}$, the function $z \rightarrow R(z: T) x$ is an $\mathfrak{X}$-valued analytic function defined on $\rho(T)$. An $\mathfrak{X}$-valued function $z \rightarrow Y(z)$ will be called an extension of $R(z: T) x$ if it is defined and analytic on an open subset $D(Y)$ of the plane and if for each $z$ in $D(Y)$, we have $(z-T) Y(z)=x$. Then, on $D(Y) \cap \rho(T)$, we have $Y(z)=R(z) x . R(z)$ is said to have the single valued extension property if for each $x$ in $\mathfrak{X}$ and each pair of extensions $Y_{1}$ and $Y_{2}$ of $R(z) x$, we have $Y_{1}=Y_{2}$ on the intersection of their domains. In this case, there exists for each $x$, a maximal extension, $R_{x}(z)$, with a domain $\rho(x)$ which contains the resolvent set of the operator.

We shall call $R_{x}(z)$ the resolvent localized at $x$. The local spectrum (at $x$ in $\mathfrak{X}$ ) is the complement of $\rho(x)$ and is denoted by $\sigma(x)$. A sufficient condition for $R(z)$ to have the single valued extension property is that $\sigma(T)$ be nowhere dense [2].

If $Y_{1}$ and $Y_{2}$ are two extensions of $R(z) x$, then for $z$ in the intersection of their domains, we have $(z-T)\left(Y_{1}(z)-Y_{2}(z)\right)=$ $x-x=0$; hence, $Y_{1}(z)-Y_{2}(z)$, if it is nonzero, is an eigenvector with eigenvalue $z$. Thus, all branching is confined to the spectrum proper. This furnishes us with another simple sufficient condition for the existence of single valued extensions; namely, the absence of the point spectrum. However, it should be noted that proper extensions may fail to exist. The positive unilateral shift on a sequential Hilbert space furnishes an example of an operator with no point 
spectrum, but no proper extension for any nonzero vector.

The following properties of the local resolvent $R_{x}(x)$ are readily established [2, p. 246].

1.1. THEOREM. The local resolvent satisfies the following:

(a) $R_{x}(z)=R(z) x$ on $\rho(T)$;

(b) $\sigma(x)$ is a compact subset of $\sigma(T)$;

(c) $\sigma(x)=\varnothing$ if and only if $x=0$;

(d) $\sigma(x+y) \subset \sigma(x) \cup \sigma(y)$;

(e) $R_{a x+b y}(z)=a R_{x}(z)+b R_{y}(z)$ on $\rho(x) \cap \rho(y)$;

(f) if $(z-T) x=0$, then $\sigma(x) \subset\{z\}$;

(g) $\sigma(T)=\bigcup\{\sigma(x): x \in \mathfrak{X}\}$,

Proof of $(f)$. For large $\lambda$,

$$
R(\lambda)=\sum_{0}^{\infty} \frac{(T-z)^{n}}{(\lambda-z)^{n+1}}
$$

thus $R_{x}(\lambda)=x /(\lambda-z)$.

Proof of $(g)$. Since we have for all $x$ in $\mathfrak{X}, \sigma(x) \subset \sigma(T)$, we assume $\lambda$ does not belong to the union of the local spectra. We have $(\lambda-T) R_{y}(\lambda) y=R_{y}(\lambda)(\lambda-T) y=y$ so that the range of $\lambda-T$ is the entire space. From (f) it is clear that $\lambda-T$ is one-to-one; thus $\lambda$ belongs to the resolvent set of the operator.

1.2. THEOREM. If $S T=T S$ for some $S$ in $B(\mathfrak{X})$, then $\sigma(S x) \subset \sigma(x)$. Moreover, if $S$ is invertible, then $\sigma(S x)=\sigma(x)$.

1.3. Theorem. (Spectral Continuity Theorem). If $T$ belongs to $B(\mathfrak{X})$ and $V$ is a neighborhood of zero in the complex plane, then there exists a $\delta>0$ such that for each $S$ in $B(\mathfrak{X})$ that commutes with $T$ and for which $|T-S| \leqq \delta$, we have $\sigma(S) \subset \sigma(T)+V$ and $\sigma(T) \subset \sigma(S)+V$.

2. The operational calculus. We will require certain restrictions on the spectrum of an operator. The family of functions, each of which is analytic on some neighborhood of the set $M$ is denoted by $\mathfrak{F}(M)$. We modify the outline of attack given in the introduction to allow a superset $M$ of the spectrum to be the base space of the function algebra. Here, by a regular function algebra, we mean a commutative semi-simple Banach algebra with unit such that for any two disjoint closed subsets of the maximal ideal space there is a function in the algebra taking the value 1 on one closed set, the 
value 0 on the other. We allow the freedom of confusing the elements of the algebra with the corresponding continuous functions on the maximal ideal space.

Definition. A subset $M$ of the complex plane is an $R$-set if $M$ is compact and nowhere dense and the uniform closure of the family $R(M)$, rational functions with poles off of $M$, is all of $C(M)$, the space of continuous functions on $M$.

If a compact nowhere dense set $M$ satisfies either of the two conditions, then it is an $R$-set [15, p. 77]:

(a) $M$ has 2-dimensional Lebesgue measure zero, or (b) the complement of $M$ has only a finite number of components.

Let $A$ be a function algebra defined on a compact nowhere dense subset $M$ of the plane $C$ (i.e., the maximal ideal space is $M$ and we identify the elements of $A$ with the continuous functions on $M$ of the Gelfand representation). We assume $A$ is regular, $\mathfrak{F}(M)$ is a dense subalgebra (in the $A$ norm), and that $M$ is an $R$-set. ${ }^{1}$ We note that under these hypothesis $A$ is inverse closed, the maximal ideal space of $A$ is $M$, and that the norm of $A$ dominates the uniform norm. Let $T$ belong to $B(\mathfrak{X})$ and have its spectrum contained in $M$. Then there exists a homomorphism $\varphi$ of $\mathfrak{F}(M)$ into $B(\mathfrak{X})$ defined by the classical operational calculus. With one additional assumption we are ready to start. Namely, we assume that $\varphi$ is bounded in the $A$ norm and so extends uniquely to a continuous homomorphism of all of $A$. We shall designate this extension by $\varphi$ also. We shall call such an algebra and mapping a pattern and a pattern calculus and denote the image of $A$ under $\varphi$ by $A(T)$. Note that $A(T)$ need not be closed in $B(\mathfrak{X})$.

Some justification of the profusion of hypotheses is afforded by the following examples which satisfy our requirements and provided the motivation for this work.

EXAMPLE A. If $U$ is a unitary operator on a Hilbert space, then $\sigma(U)$ is contained in the boundary of the unit circle, $D=\{z:|z|=1\}$, $\varphi$ extends to $C(D)$, and $A$ can be taken to be all of $C(D)$. There exists a resolution of the identity, that is, a family $\left\{E_{t}: t \in D\right\}$ of projections which commute with $U$. The ranges and null spaces of these projections form a system of closed reducing manifolds.

Since a unitary operator is an invertible isometry, $\left|U^{n}\right|=1$ for $-\infty<n \infty$. Nagy [10] proved that if an operator $T$ on a Hilbert space satisfies $\left|T^{n}\right| \leqq K$ for $-\infty<n<\infty$, then there exists a

1 The $R$-set requirement may be replaced with the assumption that $A$ is symmetric; the purpose of these assumptions is to insure that $A$ is uniformly dense in $C(M)$. In the latter case, Lemma 2.1 must be replaced with a longer construction. 
bounded invertible operator $S$ such that $S T S^{-1}$ is unitary. Moreover, if $K=1$, then $T$ is itself unitary. This leads one to hope that an operator $T$ satisfying such a growth condition on an arbitrary Banach space will enjoy a few of the pleasant properties of a unitary operator.

EXAMPLE B. If $T$ in $B(\mathfrak{X})$ satisfies $\left.\left|T^{n} \leqq K\right| n\right|^{q}$ for $n \neq 0$, we say that $T$ is of polynomial growth (of order $q$ ). In case $q=0$, then $T$ will be called an operator of bounded growth. Lorch, in [9], used the term weakly almost periodic for such an operator $(q=0)$ on a reflexive space. If $q=1$, then the operator is of a class considered by Leaf [7].

A concrete example of this class of operators is furnished by the translation operator on $L_{p}$ of the real line where the measure is given by Lebesgue measure multiplied by the absolute value of a polynomial.

If $T$ is of bounded growth, then $\sigma(T)$ is easily shown to be contained in the boundary of the unit disk. There is an obvious homomorphism of the Wiener algebra $W_{0}$ of functions on $D=\{z:|z|=1\}$ with absolutely convergent Fourier series into $B(\mathfrak{X})$ defined by

$$
\varphi: f \rightarrow f(T)=\sum c_{n} T^{n} \text { for } f(s)=\sum c_{n} \exp \text { (ins). }
$$

In [16], Wolf employed a homomorphism of $C^{2}(D)$, the class of functions on $D$ with a continuous second derivative. While $C^{2}$ is pleasanter to work with than $W_{0}$, the homomorphism in this case is not as obvious. We shall show Wolf's homomorphism and results can be obtained via a homomorphism of $W_{0}$.

An annoying point arises from the fact that a function $f$ may belong to $\mathfrak{F}(T)$ and fail to belong to $A$. For example, $f$ may have a pole on $M$ yet still be analytic on some neighborhood of $\sigma(T)$. However, if $f$ belongs to $F(T)$, it can be shown that there exists a function $g$ in $A$ with

$$
\varphi(g)=\frac{1}{2 \pi i} \int_{\gamma} f(z) R(z ; T) d z .
$$

Suppose $f$ is analytic on a neighborhood $U$ of $\sigma(T)$. Let $\sigma(T) \subset U_{1} \subset U$ and let $I$ be the ideal of functions which vanish on $U_{1}^{-} \cap M$. Since $f$ is analytic it operates on the algebra $A / I$ and hence there is a function $g$ in $A$ with $f=g$ on a neighborhood of the spectrum.

It appears that we should be considering equivalence classes of functions. If $f$ vanishes on a neighborhood of $\sigma(T)$, we shall show that $\varphi(f)=0$. In the converse direction, only this holds: if $\varphi(f)=0$. then $f$ vanishes on $\sigma(T)$. The first impression that this technical 
difficulty may be eliminated by taking the quotient algebra $W_{0} / \operatorname{Ker} \varphi$ is in error; if $\operatorname{Ker} \varphi$ is not an ideal of spectral synthesis, then $W_{0} / \operatorname{Ker} \varphi$ fails to be semi-simple.

If $T$ is of polynomial growth of order $q$, then a homomorphism of a certain subalgebra of the Wiener algebra is used to obtain an analysis of $T$.

ExAmple C. Smart [14] defined $T$ to be a well-bounded operator if there exists a homomorphism of the absolutely continuous functions on the interval $I=[a, b]$ which sends $f(s)=s$ into $T$ and which is bounded under the norm

$$
|f|=\sup \{|f(s)|: s \in I\}+\operatorname{var}\{f(s): s \in I\} .
$$

Hence a pattern calculus based on the absolutely continuous functions on $I$ can be defined. Actually, Smart included in his definition only such operators on a reflexive space; we shall employ the term with the underlying space arbitrary.

A well-bounded operator on a reflexive space has a strongly continuous finitely additive resolution of the identity [14]. In this case, an operational calculus, also based on the absolutely continuous functions, can be built from an operator valued Riemann integral in the strong operator topology. Ringrose [13] proved that a well-bounded operator on a reflexive space is "scalar" in the sense that if we set

$$
S=\int_{a}^{b} t d E(t),
$$

then $T-S=0$.

EXAmple D. Dunford [2] showed that if $R$ is an algebra of bounded operators on a reflexive Banach space $\mathfrak{X}$ which is equivalent to $C(M)$, then every operator in $R$ is a scalar type spectral operator of class $\mathfrak{X}^{*}$. Here $A$ can be taken to be $C(M)$ and $A(T)=R$.

We shall have more to say concerning Examples $\mathrm{B}$ and $\mathrm{C}$.

The development that follows depends on the Extension Theorem 2.2 below. It is here (and only here) that we employ the $R$-set property of $M$. Hence, this assumption could be dispensed with if the assertion were to be hypothesized outright.

2.1. Lemma. Let $f$ belong to a pattern $A$. Then there exists $g$ in $A$ which never vanishes and approximates $f$ uniformly.

Proof. Since the algebra is based on an $R$-set, we can approximate $f$ by $p / q$ where $p$ and $q$ are polynomials. Since $p$ can vanish 
only at a finite number of points, we can replace $p$ by $p_{1}$ which does not vanish on $M$. Now we use the $R$-set property again to uniformly approximate $p_{1} / q$ with $g$ in $A$.

2.2. Theorem. (Extension Theorem) Let $K$ be a closed subset of $M$ and $A$ be a pattern defined on $M$. Let $f$ be a function in $A$ which does not vanish on $K$. Then there exists a function $g$ in $A$ which agrees with $f$ on a neighborhood of $K$ and is non-vanishing on $M$.

Proof. With no loss of generality, we may assume $U$ is a neighborhood of $K$ with $|f| \geqq 1$ on $U$. By regularity, there exists $h$ in $A$ with $h=1$ if $|f| \geqq 1$ and $h=0$ if $|f| \leqq \frac{1}{2}$. By the lemma, there exists $g$ in $A$, never vanishing, and approximating $f$ with $|f-g| \leqq(2 \max |h|+1)^{-1}$. Let

$$
f_{1}=h f+(1-h) g=f+(1-h)(f-g) .
$$

Then $f_{1}$ is the required function in $A$.

For $f$ belonging to $A$, we define $S_{f}=\{t \in M: f(t) \neq 0\}$ and let $C l$ denote the closure operation.

2.3. Theorem. (Spectral Identification Theorem) Let $A$ be $a$ pattern algebra for the operator $T$. Then

$$
C l\left[\sigma(x) \cap S_{f}\right] \subset \sigma(f(T) x) \subset \sigma(x) \cap C l S_{f} .
$$

Proof. Set $g_{\lambda}(t)=f(t) /(\lambda-t)$. Then $g_{\lambda}$ belongs to $A$ for $\lambda$ belonging to the complement of $C l S_{f}$. This is shown by the following argument. By the Extension Theorem 2.2, there is an extension of the function $\lambda-t$ that is non-vanishing in a neighborhood of $\lambda$ which is contained in the interior of the zero set of $f$. This extension is non-vanishing on $M$, so is invertible in $A$ by the WienerGelfand Theorem. This inverse, when multiplied by $f$, is precisely $g_{\lambda}$. Hence, $\varphi\left(g_{\lambda}\right) x=G(\lambda) x$ is $\mathfrak{X}$-valued analytic since $g_{\lambda}$ is analytic in $A$ and satisfies $(\lambda-T) G(\lambda) x=f(T) x$, and so it is an extension of the resolvent at $f(T) x$. Therefore $\sigma(f(T) x) \subset C l S_{f}$ which, together with Theorem $1.1(\mathrm{~b})$, implies that

$$
\sigma(f(T) x) \subset \sigma(x) \cap C l S_{f} .
$$

We now use this result to establish the other half of the assertion. If $\lambda$ is in $\delta(x)$ and $f(\lambda) \neq 0$, then there exists a neighborhood $V$ of $\lambda$ and a function $g$ in $A$ such that $f+g$ never vanishes while $g$ is identically zero on $V$. Then 


$$
\lambda \in \sigma(x)=\sigma(\varphi(f+g) x)
$$

since $\varphi(f+g)$ is invertible in $B(\mathfrak{X})$. Moreover,

$$
\sigma(\varphi(f+g) x) \subset \sigma(f(T) x) \cup \sigma(g(T) x)
$$

by Theorem $1.1(\mathrm{~d})$. But

$$
\sigma(\varphi(g) x) \subset \sigma(x) \cap C l S_{g}
$$

while $\lambda$ does not belong to $C l S_{g}$, so we must have $\lambda \in \sigma(\varphi(f) x)$. Thus

$$
\sigma(x) \cap S_{f} \subset \sigma(f(T) x) .
$$

Since $\sigma(f(T) x)$ is closed, this implies the result.

2.4. Corollary. Suppose that $f$ belongs to $A$ :

(a) if $f$ vanishes on a neighborhood of $\sigma(T)$, then $f(T)=0$;

(b) if $f(T) \mathfrak{X}=0$, then $f$ vanishes on $\sigma(T)$.

(c) if $f$ vanishes on a neighborhood of $\sigma(x)$, then $f(T) x=0$;

(d) if $f(T) x=0$, then $f$ vanishes on $\sigma(x)$;

2.5. Theorem. (Spectral Mapping Theorem) For $f$ in $A, \sigma(f(T))=$ $f[\sigma(T)]$

Proof. Let $\left[f_{n}\right\}$ be a sequence of functions in $\mathfrak{F}(M)$ that converges to $f$. The standard Spectral Mapping Theorem $[4$, p. 569] implies that for each $n, \sigma\left(f_{n}(T)\right)=f_{n}[\sigma(T)]$. The Spectral Continuity Theorem 1.3 implies that for each neighborhood $V$ of the point 0 in the complex plane, when $n$ is sufficiently large, we have

$$
\sigma\left(\varphi\left(f_{n}\right)\right) \subset \sigma(\varphi(f))+V \text {, and } \sigma(\varphi(f)) \subset \sigma\left(\varphi\left(f_{n}\right)\right)+V .
$$

We then have ultimately

$$
f_{n}[\sigma(T)] \subset \sigma(f(T))+V \text {, and } \sigma\left(f(T) \subset f_{n}[\sigma(T)]+V .\right.
$$

The norm convergence of $\left\{f_{n}\right\}$ to $f$ implies uniform convergence which, together with the fact that the spectrum is always compact, yields the desired result.

We know by Corollary 2.4 (b) that if $f$ is $\operatorname{in} \operatorname{Ker} \varphi$, then $f$ vanishes on $\sigma(T)$. The converse fails to be true in the general. If $f$ vanishes on $\sigma(T)$, we know $|\sigma(f(T))|=0$, but the spectral radius is only dominated by the norm and is not necessarily equivalent. Hence $\sigma(f(T))=[\{0\}$ does not imply that $f(T)$ is the zero operator An operator may behave in a quasi-nilpotent or nilpotent manner on a particular manifold; for this reasons, equality is not to be expected in Theorem 2.3. 
2.6. Corollary. For $f$ in $A$, the operator $f(T)$ is quasinilpotent if and only if $f[\sigma(T)]=\{0\}$.

2.7. CoRollary. The algebra $A(T)$ is inverse closed in the sense that if $F$ belongs to $A(T)$ and has an inverse $G$ in $B(\mathfrak{X})$, then $G$ belongs to $A(T)$.

Proof. $F$ is the uniform limit of analytic functions of the operator. Since $F$ is invertible in $B(\mathfrak{X})$ this sequence must be ultimately invertible. Continuity of inversion completes the proof.

We denote the norm closure of $A(T)$ by $A(T)^{-}$; then the commutivity of $A(T)^{-}$, together with the fact that $A(T)$ is inverse closed, implies that $A(T)^{-}$is also inverse closed. Any norm closed inverse closed algebra containing $I$ and $T$ must contain all rational functions of $I$ and $T$. Hence, $A(T)^{-}$is the minimal norm closed algebra over $I$ and $T$ which contains the inverses of all its invertible members. This is known as the full algebra of $T$ [3]. If $\varphi$ is oneto-one and $\varphi(A)$ is closed then $\varphi$ is continuous in the uniform norm on $A$. See Example D concerning this case.

Definition. The hull of an ideal $I$, denoted by $H(I)$, is the collection of all maximal ideals which contain $I$. For any collection $S$ of maximal ideals, the kernel, $K(S)$, is the intersection of maximal ideals belonging to $S$. The following facts are basic [8].

(a) If $S \subset M$, then $K(S)$ is a closed ideal since it is the intersection of closed ideals.

(b) By regularity of $A$, each closed subset of $M$ is a hull. In fact, if $S \subset M$, then $S^{-}=H K(S)$.

(c) For each ideal $I$ in $A, H(I)^{-}=H(I)$.

(d) For each ideal $I$ in $A, I^{-} \subset K H(I)$. Note that the inclusion in (d) need not be equality. An ideal $I$ for which $I=K H(I)$ is called an ideal of spectral synthesis. Clearly, any such ideal is closed. We call a hull a hull of spectral synthesis if the closure of the ideal of functions in $A$, each of which vanishes in some neighborhood of the hull, is the ideal of all functions of the algebra which vanish on the hull.

2.8. Corollary. $H(\operatorname{Ker} \varphi)=\sigma(T)$.

2.9. Corollary. Let Rad $A(T)$ denote the ideal of quasinilpotent elements of $A(T)$. Then $\operatorname{Rad} A(T)=\varphi[K(\sigma(T))]$.

2.10. CoRollary. If $\sigma(T)$ is a set of spectral synthesis, then $\operatorname{Rad} A(T)=0$. 
Proof. Since $\sigma(T)$ is of spectral synthesis, any function vanishing on $\sigma(T)$ may be approximated by a function vanishing in a neighborhood of $\sigma(T)$ hence is in the closure of the closed ideal $\operatorname{Ker} \varphi$.

In a like manner, if $\sigma(x)$ is a hull of spectral synthesis and if $f$ vanishes on $\sigma(x)$, then $f(T) x=0$.

2.11. Theorem. (Closure Property) Let $K$ be closed in $M$ and let $\mathfrak{X}_{K}$ be the manifold of $x \in \mathfrak{X}$ such that $\sigma(x) \subset K$. Then $\mathfrak{X}_{K}$ is a closed subspace of $\mathfrak{X}$.

Proof. Suppose $\left\{x_{n}\right\}$ is a sequence in $\mathfrak{X}_{k}$ which converges to $x$. If $f$ vanishes on a neighborhood of $K$, then $f(T) x_{n}=0$ and, by continuity of $f(T)$, also $f(T) x=0$. Hence, $f$ vanishes on $\sigma(x)$. If $\sigma(x)$ is not a subset of $K$, then regularity of $A$ furnishes a function $f$ vanishing on $K$ but not on all of $\sigma(x)$.

Non-trivial manifolds are generated by proper closed subsets of $\sigma(T)$. Such manifolds are invariant under $T$, and, moreover, invariant under every operator in $A(T)$ since

$$
\sigma(f(T) x) \subset \sigma(x) \cap C l S_{f} \subset \sigma(x) \subset K
$$

for $f$ in $A$.

2.12. Theorem. Let $K$ and $N$ be closed in $M$. Then

(a) $\mathfrak{X}_{K} \cap \mathfrak{X}_{N}=\mathfrak{X}_{K \cap N}$;

(b) $\left(\mathfrak{X}_{K}+\mathfrak{X}_{N}\right)^{-} \subset \mathfrak{X}_{K \cup N}$;

(c) if $K \cap N=\varnothing$, then $\mathfrak{X}_{K \cup N}=\mathfrak{X}_{K}+\mathfrak{X}_{N}$ so that $\mathfrak{X}_{K}+\mathfrak{X}_{N}$ is closed.

Proof.

(a) Trivial.

(b) If $x=w+z$ with $\sigma(w) \subset K$ and $\sigma(z) \subset N$, then, since $\sigma(w+z) \subset \sigma(w) \cup \sigma(z)$, we have $\sigma(x) \subset K \cup N$ so $\mathfrak{X}_{K}+\mathfrak{X}_{N} \cup \mathfrak{X}_{K \cup N}$. The assertion follows from monotonicity of the closure operation. (c) If $\sigma(x) \subset K \cup N$, then $x=w+z$ where $\sigma(w) \subset K$ and $\sigma(z) \subset N$ by regularity of $A$ and the fact that $K \cap N=\varnothing$.

2.13. TheOREM. (Inclusion Property) If $K$ is closed in $M$, then the spectrum of the restriction of $T$ to the closed manifold $\mathfrak{X}_{K}$ is contained in $K$.

Proof. If $\lambda$ does not belong to $K$, then there exists a function $f$ in $A$ with $f(z)=1 /(\lambda-z)$ on a neighborhood of $K$. If $x \in \mathfrak{X}_{K}$, then we have $(\lambda-T) f(T) x=f(T)(\lambda-T) x=x$. Hence $\lambda$ is in the resolvent set of the restriction. 
3. The $N^{*}$-algebra. In this section, we shall examine the implications of an additional hypothesis on the pattern algebra. This is the $N^{*}$-condition to be defined below. The application is to operators of bounded growth and, since the same techniques apply to operators of polynomial growth, we treat them also in this section although the $N^{*}$-condition is not satisfied in general.

Definition. Recall that an ideal $I$ is of spectral synthesis if $K H(I)=I$. An algebra in which each closed ideal with a one-point hull is an ideal of spectral synthesis is called an $N^{*}$ algebra [11, p. 92].

For a pattern $A$, this means the following: if $t$ is a point of $M$ and $f$ a function in $A$ which vanishes at $t$, then for each $\in>0$, there is a function $g$ in $A$ which vanishes in a neighborhood of $t$ and such that $|f-g| \leqq \varepsilon$.

3.1. TheoREM. (Lorch Approximation) Let $T$ be an operator with an $N^{*}$-pattern. For each $\varepsilon>0$ and each $z$ in $\sigma(T)$, there is $a \delta>0$ such that $|(T-z) x| \leqq \varepsilon K|x|$ for vectors $x$ with $\sigma(x)$ contained in the neighborhood $N_{\delta}(z)$ of radius $\delta$ about the point $z$.

Proof. The function $f(t)=t-z$ vanishes at $t=z$. Hence, by the $N^{*}$-condition, there exists a function $g$ with the properties that $|f-g| \leqq \varepsilon$ and $g$ vanishes in some neighborhood of $t=z$. Then

$$
|(T-z) x|=|\varphi(f) x| \leqq|\varphi(f-g) x|+|\varphi(g) x| \leqq \varepsilon K|x|+|\varphi(g) x| .
$$

Now, if $\sigma(x)$ is disjoint from the closed support of $g$, then $\varphi(g) x=0$ by Corollary 2.4 (c). The assertion then obtains if $\sigma(x) \subset N_{\delta}(z)$ for a small enough $\delta<0$.

Lorch [9] obtained this result for operators of bounded growth. We will obtain this result as a corollary of the fact that $W_{0}$ is an $N^{*}$-algebra and it will be clear that in this case $\delta$ can be taken independently of $z$. We indicate a further generalization of the theorem in the following direction. Define an $N^{*}(q)$-algebra by the condition that $f(z)=0$ implies that $(f)^{q}$ can be approximated in the algebra by functions, each of which vanishes in some neighborhood of $z$. Then, if $T$ has an $N^{*}(q)$-pattern, it follows from a similar argument that for $z$ in $\sigma(T)$ and $\varepsilon>0$, there exists a $\delta<0$ such that $\left|(T-z)^{p} x\right| \leqq \varepsilon K|x|$ for those vectors $x$ with $\sigma(x) \subset N_{\delta}(z)$. We shall obtain this last result for operators of $q$ th order growth by a study of the ideals with one point hulls in the pattern algebra.

3.2. Theorem. (Density Theorem) Let $A$ be an $N^{*}$-pattern 
algebra for the operator $T$ and let $z$ belong to the continuous spectrum. Then

$$
\mathfrak{X}(z)=\left\{x \in \mathfrak{X}: z \in \in^{\prime} \sigma(x)\right\}
$$

is dense in $\mathfrak{X}$.

Proof. Since $z$ belongs to the continuous spectrum, we know $(z-T) \mathfrak{X}$ is dense in $\mathfrak{X}$. Denote by $J(z)$ the ideal of functions in $A$, each of which vanishes in some neighborhood of the point $z$. Let $\left\{f_{n}\right\}$ belong to $J(z)$ and converge to $g(t)=t-z$. For $x$ in $\mathfrak{X}$, there exists a sequence of vectors $\left\{w_{n}\right\}$ such that $\varphi(g) w_{n}=y_{n}$ converges to $x$. Given $\varepsilon>0$, choose $w_{n}$ so that $\left|\varphi(g) w_{n}-x\right| \leqq \varepsilon$. Then choose $f_{k}$ in $J(z)$ so that $\left|\varphi\left(f_{k}\right) w_{n}-\varphi(g) w_{n}\right| \leqq \varepsilon$. Then

$$
\left|\varphi\left(f_{k}\right) w_{n}-x\right| \leqq\left|\varphi\left(f_{k}\right) w_{n}-\varphi(g) w_{n}\right|+\left|\varphi(g) w_{n}-x\right| \leqq 2 \varepsilon,
$$

where $\sigma\left(\varphi\left(f_{k}\right) w_{n}\right)$ is disjoint from $\{z\}$.

If $\left\{z_{n}\right\}$ belongs to the continuous spectrum for $n=1, \cdots, k$, then, in a similar fashion, it can be shown that

$$
\mathfrak{X}\left(z_{1}, \cdots, z_{k}\right)=\left\{x \in \mathfrak{X}: z_{n} \in{ }^{\prime} \sigma(x), n=1, \cdots, k\right\}
$$

is dense in $\mathfrak{X}$.

If the spectrum of $T$, an operator with an $N^{*}$-pattern, is a finite union of simple arcs, then the possibility of a decomposition of the operator with respect to points in the continuous spectrum arises. For notational convenience, we assume $\sigma(T)$ is contained in the unit interval $[0,1]$. Then, if $t$ is a point in the continuous spectrum, a projection $P(t)$ associated with the interval $[0, t]$ can be defined on the dense manifold $\mathfrak{X}(t)$. For, if $x$ is in $\mathfrak{X}(t)$, then, by regularity of $A$, we can write $x=u+w$ with $\sigma(u)$ contained in the interval $[0, t)$ and $\sigma(w)$ contained in the interval $(t, 1]$. The projection is defined by $P(t) x=u$. This projection has a closed extension.

3.3. THEOREM. Let $T$, an operator with an $N^{*}$-pattern, have a a purely continuous spectrum which is contained in the unit interval. Then, for each point $t$ in the unit interval, there is a densely defined projection $P(t)$ which has a closed extension.

Proof. The preceding discussion shows the existence of $P(t)$ defined on $\mathfrak{X}(t)$. The closure of the graph of $P(t)$ is the closed extension provided this closure is an operator. Hence, the assertion is verified once we show that if $x_{n} \in \mathfrak{X}(t)$ with $x_{n} \rightarrow 0$, and if $P(t) x_{n}=$ $y_{n} \rightarrow y_{0}$, then $y_{0}=0$. We know $\sigma\left(y_{n}\right) \subset[0, t]$ and, since $x_{n}=$ $w_{n}+y_{n}$, that $w_{n} \rightarrow-y_{0}$. But $\sigma\left(w_{n}\right) \subset[t, 1]$ so $\sigma\left(-y_{0}\right)=\sigma\left(y_{0}\right) \subset\{t\}$. 
Hence, if $y_{0} \neq 0$, we have $\sigma\left(y_{0}\right)=\{t\}$. By Theorem 3.1, $\left|(T-t) y_{0}\right|=0$ which contradicts the assumption that $t$ is not in the point spectrum. Hence, $P(t)$ has a closed extension which we shall denote also by $P(t)$.

For an operator of polynomial growth, we shall construct a calculus based on $W_{0}$ or a certain subalgebra. We shall examine these algebras before applying the theory to operators.

Let $D$ denote the boundary of the unit circle and $W_{0}$ the algebra of functions defined on $D$ which have absolutely convergent Fourier series. For $f$ in $W_{0}, f(t)=\sum c_{n} \exp$ (int), we define $|f|_{0}=\sum\left|c_{n}\right|$. Then $W_{0}$ is a semi-simple symmetric commutative Banach algebra with a unit under point-wise operations and which has $D$, under the usual topology, as the space of maximal ideals.

Definition. For $q=0,1, \cdots$ we define $W_{q}$ to be the subalgebra of $W_{0}$ consisting of those functions $f(t)=\sum c_{n} \exp$ (int) for which $\sum\left|c_{n} n^{q}\right|<\infty$. Then $W_{q}$ is a Banach algebra in its own right under the norm

$$
|f|_{q}=\left|c_{0}\right|+\sum\left|c_{n} n^{q}\right| \text {. }
$$

It is well known that $W_{q}$ is a semi-simple Banach algebra with maximal ideal space $D$ under the usual topology [11, p. 299]. We summarize in the following lemma the properties of $W_{q}$ pertinent to our argument [17].

\section{LEMMA 3.4.}

(a) If $f$ has a continuous derivative of order $q+2$, then $f^{(k)}$ belongs to $W_{0}$ for $k=0, \cdots, q$.

(b) If $f^{(q+2)}$ exists as a continuous function, then $f$ belongs to $W_{q}$. Conversely, if $f$ belongs to $W_{q}$, then $f^{(q)}$ exists and is continuous.

(c) If a function $f(t)=\sum c_{n} \exp$ (int) belongs to $W_{q}$, then $f^{(k)}(t)=$ $\sum c_{n}(\text { in })^{k} \exp$ (int) for $k=0, \cdots, q$ and $|f|^{q}=\left|f^{(k)}\right|_{q-k}$.

(d) For $f$ in $W_{q}, f(t)=\sum c_{n} \exp$ (int), define $f_{k}(t)=\sum_{-k}^{k} c_{n} \exp$ (int). Then $f_{k}$ converges to $f$ in the $W_{q}$ norm (and therefore also uniformly). It follows that $W_{q}$ is a regular algebra and the class $\mathfrak{F}(D)$ is dense in $W_{q}$.

3.5. Theorem. (Helson-Silov) Let $I$ be a closed ideal in the $L_{1}$ algebra of a locally compact Abelian group and let $f$ be a function in $L_{1}$ such that $H(I)$ is contained in $H(f)$. If that part of the boundary of $H(f)$ which is included in $H(I)$ contains no nonvoid perfect set, then $f$ belongs to $I$.

The proof is to be found in $[8, \mathrm{p} .151]$. Since $W_{0}$ is isometrically 
isomorphic to the space $L_{1}$ of the integers, it follows from the above theorem that $W_{0}$ is an $N^{*}$-algebra.

The $N^{*}$-condition does not hold for $W_{q}$ for $q \neq 0$. We will, however, want to answer to question: what functions are in the closure of the ideal $J\left(\lambda_{0}\right)$ of functions of $W_{q}$, each of which vanishes in some neighborhood of the point $\lambda_{0}$ ?

First, if $f$ has zero constant term, it has a $k$ th integral which is given by the formal series except for a possible constant term of the integral. Now suppose $\left\{f_{i}\right\}$ is a sequence of functions in $J\left(\lambda_{0}\right)$ which converges to $g$ in the $W_{q}$ norm. Then $f_{i}^{(k)} \rightarrow g^{(k)}$ in the $W_{q-k}$ norm for $k=0, \cdots, q$. Since norm convergence in a function algebra implies uniform convergence on the maximal ideal space, it is clear that $g^{(k)}\left(\lambda_{0}\right)=0$ for $k=0, \cdots, q$. We assert that this condition is also sufficient. The proof is inductive and can be outlined as follows.

With no loss of generality, we may take $\lambda_{0}=1$. If $g$ belongs to $W_{1}$ with $g(1)=g^{(1)}(1)=0$, then $g^{(1)}$ belongs to $\mathrm{W}_{0}$ and vanishes at the point 1 . By the $N^{*}$ condition in $W_{0}$, there exists a sequence of functions $\left\{f_{i}\right\}$ in $W_{0}$ which converges to $g^{(1)}$ in $W_{0}$ norm and each function vanishes in some neighborhood of the point 1 . Since $g^{(1)}$ has zero constant term, we may and do take the functions $f_{i}$ to have zero constant terms. This normalization may be carried out as follows. Choose $h$ in $W_{0}$ with constant term 1 and such that $h$ vanishes in a neighborhood of the point 1 . The normalized approximating sequence is given by

$$
f_{i}(t)-h(t)\left[\frac{1}{2 \pi} \int_{0}^{3 \pi}\left(f_{i}-g^{(1)}\right)(s) d s\right]
$$

We now change notation to let $\left\{f_{i}\right\}$ denote the approximating sequence with zero constant terms. We may now integrate back up to $W_{1}$ to obtain a sequence of functions, each of which vanishes in a neighborhood of the point 1 . This sequence converges to $g$ since the norm of the difference is given by the $W_{0}$ norm of the difference of unintegrated function except for a possible constant term which must tend to zero since $\left\{f_{i}\right\}$ converges uniformly to $g^{(1)}$. The inductive step is essentially a repetition of this same argument. Thus we have the following.

3.6. THEOREM. The closure of the ideal $J\left(\lambda_{0}\right)=\left\{f \in W_{q}: f\right.$ vanishes in some neighborhood of $\lambda_{0}$ \} is given by

$$
J\left(\lambda_{0}\right)^{-}=\left\{h \in W_{q}: h^{k}\left(\lambda_{0}\right)=0 \text { for } k=0, \cdots, q\right\} .
$$

Observe that the proof is valid with $\lambda_{0}$ replaced by any closed set $K$ which is a hull of spectral synthesis in $W_{0}$ For example, if 
the set $K$ is the closure of its interior, and if $f$ vanishes on $K$, then $\partial H(f) \cap H(I)$ is countable and so can contain no nonvoid perfect set.

Recall that $T$ is said to have polynomial growth of order $q$ if, for some constant $K$ and all nonzero integers $n$, we have

$$
\left|T^{n}\right| \leqq K\left|n^{q}\right|
$$

It is easily shown that if $T$ satisfies $(*)$, then $\sigma(T)$ is contained in the boundary of the unit circle. Also $(*)$ implies that for $|z| \neq 1$,

$$
|R(z)| \leqq \frac{1}{\left.|1-| z\right|^{q+1}} .
$$

Conversely, if $(* *)$ holds then $T$ is of polynomial growth of order $q+1[16]$.

For $T$ of $q$ th order growth, we define the operational calculus on $W_{q}$ as follows: for $f(t)=\sum c_{n} \exp$ (int) in $W_{q}$, we set $\varphi(f)=f(T)=$ $\sum c_{n} T^{n}$. It follows at once that $\varphi$ is a continuous homomorphism into an algebra of operators.

Since $W_{0}$ is an $N^{*}$-algebra, the Lorch Approximation is valid for every operator of bounded growth and it is clear that this approximation is uniform for $z$ in $\sigma(T)$. We shall obtain the generalization of the Lorch Approximation for operators of polynomial growth.

3.7. THeOREM. Let $T$ be of qth order growth. Given $\in>0$, there exists $\delta>0$ such that for $z$ in $\sigma(T)$, we have $\left|(T-z)^{q} x\right| \leqq$ $\varepsilon K|x|$ for $x$ with $\sigma(x)$ in $N_{\delta}(z)$.

Proof. We let $g(t)=(\exp (i t)-z)^{q}$. From Theorem 3.6, there exists a function $f$ vanishing on a neighborhood of $z$ such that $|f-g|_{q}<\varepsilon$. So

$$
\left|(T-z)^{q} x\right| \leqq|g-f|_{q}|x|+|\varphi(f) x| \cdot
$$

Now, if $\sigma(x)$ is disjoint from the closed support of $f$, the conclusion obtains.

We now give indications of an estimate of the Lorch Approximation for operators of bounded growth. We know that if $T$ is of bounded growth, then for $|z| \neq 1$ we have

$$
|R(z)| \leqq \frac{M}{|1-| z||}
$$

The complement of $\sigma(T)$ is open in $D$. Let $(a, b)$ denote an open arc of the complement. Pick $a_{1}$ and $b_{1}$ in this open arc and let $L\left(a_{1}\right)$ 
and $L\left(b_{1}\right)$ be the radial lines through $a_{1}$ and $b_{1}$ respectively. Set

$$
G(z)=R(z)\left(z-a_{1}\right)\left(z-b_{1}\right)(z-c)^{-1}
$$

where $c$ is the midpoint of the arc complementary to $(a, b)$. Then $G(z)$ is an analytic operator valued function in the wedge determined by $L\left(a_{1}\right)$ and $L\left(b_{1}\right)$.

On $L\left(a_{1}\right)$ we have the following estimate:

$$
|G(z)| \leqq M \frac{\left|z-b_{1}\right|}{|z-c|} \leqq M \frac{|z-c|+\left|c-b_{1}\right|}{|z-c|} \leqq 3 M .
$$

This same estimate is valid on $L\left(b_{1}\right)$. Since $G(z) \rightarrow I$ as $s \rightarrow \infty$, we have $|G(z)| \leqq 3 M$ throughout the wedge by the maximum modulus theorem. Hence, on the wedge determined by the radial lines through $a$ and $b$, we have

$$
|R(z)| \leqq 3 M\left|(z-c)(z-a)^{-1}(z-b)^{-1}\right| \text {. }
$$

This inequality has a local version also; the same argument applied to $R_{x}(z)$ yields

$$
\left|R_{x}(z)\right| \leqq 3 M|x|\left|(z-c)(z-a)^{-1}(z-b)^{-1}\right|
$$

in the wedge determined by the arc $(a, b)$ of the complement of $\sigma(x)$. Using this inequality to estimate

$$
\left|\frac{1}{2 \pi i} \int_{\gamma}\left(z-\lambda_{0}\right) R_{x}(z) d z\right|,
$$

we find that $\left|\left(T-\lambda_{0}\right) x\right| \leqq 6 M|x| d(a, b)$ for $x$ with spectrum in the arc $(a, b)$ where $d(a, b)$ denotes the curvilinear arc length of $(a, b)$.

Wolf [17] demonstrated, for $T$ of $q$ th order growth, the existence of a bounded homomorphism of $C^{q+2}(D)$, the functions on $D$ with a continuous derivative of order $q+2$, sending $f(t)=t$ into $T$. We find it simpler to obtain this homomorphism in the following manner. First, we know by Lemma 3.4 (b) that $C^{q+2}$ is contained in $W_{q}$. The mapping $C^{q+2} \rightarrow W_{q}$ is shown to be continuous as follows. If $f$ is in $C^{q+2}$ with norm in this algebra bounded by $B$, then the Fourier coefficients of $f^{(q+2)}$ are bounded by $B$. Hence the Fourier coefficients of $f^{(q)}$ are summable and $\left|f^{(q)}\right|_{0}=|f|_{q}+\left|c_{0}\right|$ is dominated by

$$
B\left(1+\Sigma^{\prime} n^{-2}\right)+\left|c_{0}\right| \leqq B\left(2+\Sigma^{\prime} n^{-2}\right),
$$

where the prime indicates omition of the constant term. Thus the composition of this mapping with the homomorphism of $W_{q}$ previously introduced is a continuous homomorphism of $C^{q+2}(D)$.

In his treatment of weakly almost periodic operators on a re- 
flexive space, Lorch [9] obtained a family of "reducing" manifolds. Under his hypotheses, Lorch proved that the residual spectrum is vacuous. We are able to obtain these manifolds by the present methods for an operator of bounded growth on an arbitrary space provided the spectrum is purely continuous. Moreover, we are able to abtain these quasi-reducing manifolds for an operator with an $N^{*}$-pattern provided the spectrum is purely continuous and is contained in a finite union of simple curves which, for simplicity, is taken to be the unit interval.

3.8. THEOREM. Let $T$ have a purely continuous spectrum which is contained in the unit interval $[0,1]$ and let $T$ have an $N^{*}$-pattern. Then there exists a family $\{P(t): 0 \leqq t \leqq 1\}$ of closed projections with $P(t)$ defined on the dense manifold $\mathfrak{X}(t)$ and possessing the following attributes:

(a) $P(0)=0$ and $P(1)=I$;

(b) $P(t) T=T P(t)$ on $\mathfrak{X}(t)$;

(c) if $s \leqq t$, then $P(s) P(t)=P(t) P(s)=P(s)$ on $\mathfrak{X}(s, t)$;

(d) when restricted to the closure of $P(t) \mathfrak{X}(t)$, $T$ has spectrum contained in $[0, t]$.

In fact, the range of $P(t)$ is $\mathfrak{X}[0, t]=\{x \in \mathfrak{X}: \sigma(x) \subset[0, t]\}$. The domain is $\mathfrak{X}[0, t] \oplus \mathfrak{X}[t, 1]$ which is dense in $\mathfrak{X}$, but not necessarily all of $\mathfrak{X}$. If $\mathfrak{X}[0, t]$ and $\mathfrak{X}[t, 1]$ are at a positive angle, then the manifolds reduce $T$ in the proper sense and the associated projection is bounded.

4. Integral representations. In Theorem 3.8 we gave a spectral decomposition for an operator that possesses an $N^{*}$-pattern algebra. We obtain, in this section, an integral representation under the additional hypothesis that the family of densely defined projections of Theorem 3.8 is uniformly bounded. We operate, as before, under the assumption that $T$ has an $N^{*}$-pattern algebra and a purely continuous spectrum contained in the unit interval. We now assume each projection has a bounded extension to all of $\mathfrak{X}$, and that the family is uniformly bounded; that is $|P(t)| \leqq K_{1}$ for each point $t$ in the unit interval. The existence of a uniform bound implies continuity of the spectral family in the strong operator topology.

4.1. THEOREM. Let $T$ have an $N^{*}$-pattern algebra, purely continuous spectrum contained in the unit interval, and a uniformly bounded spectral family of projections. Then $P(t)$ is continuous in the strong operator topology.

Proof. It must be shown that 


$$
\lim _{s \rightarrow t}|P(t) x-P(s) x|=0
$$

for each $x$ in $\mathfrak{X}$. This clearly holds for $y$ in $\mathfrak{X}(t)$; in this case, $P(t) y-P(s) y=0$ whenever $s$ is close enough to $t$. For $x$ in $\mathfrak{X}$, we choose $y$ in $\mathfrak{X}(t)$ to approximate $x$ to within $\varepsilon$ and obtain

$$
\begin{aligned}
|P(t) x-P(s) x| & \leqq|P(t)-P(s)||x-y|+|P(t) y-P(s) y| \\
& \leqq 2 K_{1} \varepsilon+|P(t) y-P(s) y| .
\end{aligned}
$$

Since the last term tends to zero, this proves the assertion.

For a point $t$ in the closed unit interval and for each $x$ in $\mathfrak{X}$, one can write $x=y+w$ with $y \in \mathfrak{X}[0, t]=M(t)$, and $w \in \mathfrak{X}[t, 1]=N(t)$. The null space of $P(t)$ is $N(t)$ and the range is $M(t)$. The manifolds $M(t)$ and $N(t)$ are closed and disjoint for if $\sigma(x) \subset\{t\}$, then $x=0$ by Theorem 3.1 and the fact that the spectrum is purely continuous. Moreover, $M(t)+N(t)=\mathfrak{X}$, for if $x \in \mathfrak{X}$, then we have $x=$ $P(t) x+(I-P(t)) x$. Thus $\mathfrak{X}=M(t) \oplus N(t)$; the manifolds reduce the the operator in the proper sense.

If $f$ is a function of bounded variation on the unit interval, then, since $t \rightarrow P(t)$ is strongly continuous, a Riemann integral of $f$ with respect to $P$ can be defined in the strong operator topology [6, p. 66].

We define

$$
S=\int t d P(t)
$$

The question which presents itself naturally is-will the integral defined above generate a calculus which agrees with the original calculus? That is, for $S$ defined above, can we claim $T-S=0$ ?

\subsection{THEOREM. $T-S$ is quasi-nilpotent.}

Proof. (The proof is patterned after a proof given by Smart [14, Theorem D (ii)] for well-bounded operators.) For $\varepsilon>0$, we partition the unit interval with a finite set of points so that the mesh of the partition is less than $\varepsilon$. Then, the restriction of $S$ and $T$ to the closed manifolds $\left(P\left(t_{i+1}\right)-P\left(t_{i}\right)\right) \mathfrak{X}$ have spectra in $\left[t_{i}, t_{i+1}\right]$. This is a consequence of Theorem 2.14 for the operator $T$. That this is true of $S$ also follows from the same theorem, for $S$ itself has a pattern calculus defined on the absolutely continuous functions given by the defining integral; in fact, $S$ is well-bounded, as the defining integral gives the homomorphism. Now, both $S-t_{i} I$ and $T-t_{i} I$ have spectra in $[0, \varepsilon]$ on the subspace. Since $S$ and $T$ commute, the spectral radius of $T-S$ on the subspace is at most 
$2 \varepsilon$. The space $\mathfrak{X}$ is the direct sum of the subspaces so that spectrum of $T-S$ on $\mathfrak{X}$ is the union of the spectra of its restrictions. Thus $|\sigma(T-S)| \leqq 2 \varepsilon$ which implies $\sigma(T-S)=\{0\}$. In the case $T$ itself is well-bounded and $\mathfrak{X}$ is reflexive, Ringrose [13], has shown $T-S=0$. Conversely, if $T-S=0$ with $\mathfrak{X}$ arbitrary, then $T$ is well-bounded.

The decomposition can be stated in a form analogous to Dunford's spectral operators.

4.3. TheOREM. Let $T$ be a bounded operator with purely continuous spectrum contained in the unit interval. Let there be a family $\{P(t): t \in[0,1]\}$ of commuting projections satisfying:

(a) $P(0)=0$ and $P(1)=I$;

(b) $P(t) T=T P(t)$;

(c) if $s \leqq t$, then $P(s) P(t)=P(t) P(s)=P(s)$;

(d) $\lim _{s \rightarrow t} P(s) x=P(t) x$;

(e) $|\stackrel{s \rightarrow t}{P}(t)| \leqq K_{1}$; and

(f) the restriction of $T$ to the closed manifold $P(t) \mathfrak{X}$ has spectrum in $[0, t]$.

Then $T=S+N$ where $S$ is a well-bounded operator defined by

$$
S=\int t d P(t)
$$

and $N$ is a commuting quasi-nilpotent.

Proof. The integral exists and defines a well-bounded operator [14]. The proof of Theorem 4.2 can be directly adopted to prove $T-S$ is quasi-nilpotent.

\section{BIBLIOGRAPHY}

1. W. G. Bade, On Boolean algebras of projections and algebras of operators, Trans. Amer. Math. Soc., 80 (1955), 373-392.

2. N. Dunford, A survey of the theory of spectral operators, Bull. Amer. Math. Soc., 64 (1958), 217-274.

3. — Spectral operators, Pacific J. Math., 4 (1954), 321-354.

4. N. Dunford and J. Schwartz, Linear operators, part I, Interscience, New York, 1958. 1958.

5. U. Fixman, Problems in spectral operators, Pacific J. Math., 9 (1959), 1029-1051.

6. E. Hille and R. S. Phillips, Functional analysis and semi-groups, Amer. Math. Soc. Coll., Vol. 31, Providence, 1957.

7. G. K. Leaf, A spectral theory for a class of linear operators, Doctoral dissertation, Univ. of Ill., 1961. To appear in Pacific J. Math.

8. L. H. Loomis, An introduction to abstract harmonic analysis, Van Nostrand, New York, 1953.

9. E. R. Lorch, The integral representation of weakly almost-periodic transformation in reflexive vector spaces, Trans. Amer. Math. Soc., 49 (1941), 18-40.

10. B. Sz.-Nagy, Extensions of linear transformations in Hilbert space which extend 
beyond the space, Ungar, New York, 1960.

11. C. E. Richart, General theory of Banach algebras, Van Nostrand, Princeton, 1960.

12. F. Riesz and B. Sz. Nagy, Functional analysis, Ungar, New York, 1955.

13. J. R. Ringrose, On well-bounded operators, J. Australian Math. Soc. I part 3 (1960), 334-343.

14. D. R. Smart, Conditional convergent spectral expansions, J. Australian Math. Soc. I part 3 (1960), 319-333.

15. J. Wermer, Banach algebras and analytic functions, Academic Press, New York, 1961.

16. F. Wolf, Operators in Banach spaces which admit a generalized spectral decomposition, Indag. Math., 19 (1957), 302-311.

17. A. Zygmund, Trigonometric series, second edition, University Press, Cambridge, 1959.

UNIVERSITY OF MINNESOTA. 


\section{PACIFIC JOURNAL OF MATHEMATICS}

\section{EDITORS}

Robert Osserman

Stanford University

Stanford, California

M. G. Arsove

University of Washington

Seattle 5 , Washington
J. DugundjI

University of Southern Califorma: Los Angeles 7, California

Lowell J. Paige

University of California

Los Angeles 24, California

\section{ASSOCIATE EDITORS}

E. F. BECKENBACH

B. H. NeumanN

F. WOLF

K. YosIDA

\section{SUPPORTING INSTITUTIONS}

UNIVERSITY OF BRITISH COLUMBIA

CALIFORNIA INSTITUTE OF TECHNOLOGY

UNIVERSITY OF CALIFORNIA

MONTANA STATE UNIVERSITY

UNIVERSITY OF NEVADA

NEW MEXICO STATE UNIVERSITY

OREGON STATE UNIVERSITY

UNIVERSITY OF OREGON

OSAKA UNIVERSITY

UNIVERSITY OF SOUTHERN CALIFORNIA
STANFORD UNIVERSITY

UNIVERSITY OF TOKYO

UNIVERSITY OF UTAH

WASHINGTON STATE UNIVERSITY

UNIVERSITY OF WASHINGTON

AMERICAN MATHEMATICAL SOCIETY CALIFORNIA RESEARCH CORPORATION SPACE TECHNOLOGY LABORATORIES NAVAL ORDNANCE TEST STATION 


\section{Pacific Journal of Mathematics}

\section{Vol. 14, No. 1 \\ May, 1964}

Richard Arens, Normal form for a Pfaffian .........................

Charles Vernon Coffman, Non-linear differential equations on cones in Banach

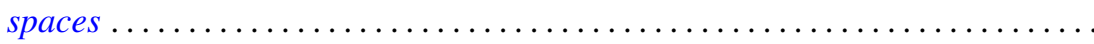

Ralph DeMarr, Order convergence in linear topological spaces ..............

Peter Larkin Duren, On the spectrum of a Toeplitz operator ................

Robert E. Edwards, Endomorphisms of function-spaces which leave stable all

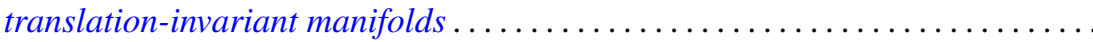

Erik Maurice Ellentuck, Infinite products of isols . . . . . . . . . . . . . . . . 49

William James Firey, Some applications of means of convex bodies . . . . . . . . 53

Haim Gaifman, Concerning measures on Boolean algebras ............. 61

Richard Carl Gilbert, Extremal spectral functions of a symmetric operator. . . . . . 75

Ronald Lewis Graham, On finite sums of reciprocals of distinct nth powers ..... 85

Hwa Suk Hahn, On the relative growth of differences of partition functions ...... 93

Isidore Isaac Hirschman, Jr., Extreme eigen values of Toeplitz forms associated

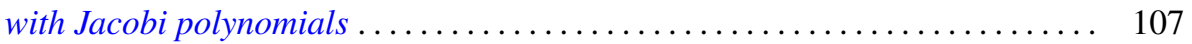

Chen-jung Hsu, Remarks on certain almost product spaces . . . . . . . . . . . 163

George Seth Innis, Jr., Some reproducing kernels for the unit disk . . . . . . . . . 177

Ronald Jacobowitz, Multiplicativity of the local Hilbert symbol . . . . . . . . . . . 187

Paul Joseph Kelly, On some mappings related to graphs ................. 191

William A. Kirk, On curvature of a metric space at a point . . . . . . . . . . . . 195

G. J. Kurowski, On the convergence of semi-discrete analytic functions . . . . . . . 199

Richard George Laatsch, Extensions of subadditive functions . . . . . . . . . . . 209

V. Marić, On some properties of solutions of $\Delta \psi+A\left(r^{2}\right) X \nabla \psi+C\left(r^{2}\right) \psi=0 \ldots 217$

William H. Mills, Polynomials with minimal value sets . . . . . . . . . . . 225

George James Minty, Jr., On the monotonicity of the gradient of a convex

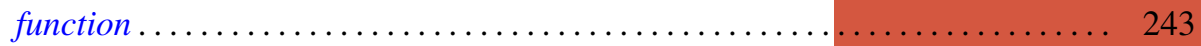

George James Minty, Jr., On the solvability of nonlinear functional equations of 'monotonic' type ................................... 249

J. B. Muskat, On the solvability of $x^{e} \equiv e(\bmod p) \ldots \ldots \ldots \ldots \ldots \ldots \ldots \ldots . \ldots \ldots$

Zeev Nehari, On an inequality of $P . R$. Bessack ................... 261

Raymond Moos Redheffer and Ernst Gabor Straus, Degenerate elliptic

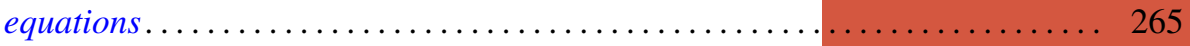

Abraham Robinson, On generalized limits and linear functionals . . . . . . . . . 269

Bernard W. Roos, On a class of singular second order differential equations with a

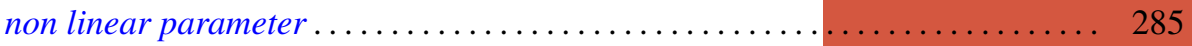

Tôru Saitô, Ordered completely regular semigroups . . . . . . . . . . . . . . . . 295

Edward Silverman, A problem of least area ....................... 309

Robert C. Sine, Spectral decomposition of a class of operators . . . . . . . . . 333

Jonathan Dean Swift, Chains and graphs of Ostrom planes . . . . . . . . . . . 353

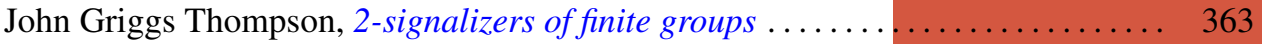

Harold Widom, On the spectrum of a Toeplitz operator . . . . . . . . . . . . . 365 